\title{
Genome-wide DNA methylation profiling of CD8+ T cells shows a distinct epigenetic signature to CD4+ $T$ cells in multiple sclerosis patients
}

Vicki E. Maltby ${ }^{1,2 \dagger}$, Moira C. Graves ${ }^{1,3 \dagger}$, Rodney A. Lea ${ }^{1,4}$, Miles C. Benton ${ }^{4}$, Katherine A. Sanders ${ }^{1,5}$, Lotti Tajouri ${ }^{5}$, Rodney J. Scott ${ }^{1,6}$ and Jeannette Lechner-Scott ${ }^{1,3,7^{*}}$

\begin{abstract}
Background: Multiple sclerosis (MS) is thought to be a T cell-mediated autoimmune disorder. MS pathogenesis is likely due to a genetic predisposition triggered by a variety of environmental factors. Epigenetics, particularly DNA methylation, provide a logical interface for environmental factors to influence the genome. In this study we aim to identify DNA methylation changes associated with MS in CD8+ T cells in 30 relapsing remitting MS patients and 28 healthy blood donors using Illumina 450K methylation arrays.
\end{abstract}

Findings: Seventy-nine differentially methylated CpGs were associated with MS. The methylation profile of CD8+ T cells was distinctive from our previously published data on CD4+ T cells in the same cohort. Most notably, there was no major $\mathrm{CpG}$ effect at the MS risk gene HLA-DRB1 locus in the CD8+ T cells.

Conclusion: CD8+ T cells and CD4+ T cells have distinct DNA methylation profiles. This case-control study highlights the importance of distinctive cell subtypes when investigating epigenetic changes in MS and other complex diseases.

Keywords: Multiple sclerosis, DNA methylation, CD8+ T cells, HLA-DRB1

\section{Findings}

Multiple sclerosis (MS) susceptibility is influenced by a combination of genetic factors and environmental exposures. CD4+ T cells have long been favoured as the most important immune cell subset in the pathogenesis of disease, but there is increasing evidence that $\mathrm{CD} 8+\mathrm{T}$ cells play a substantial role in central nervous system damage (reviewed in [1]).

Despite several large genome-wide association studies (GWAS), there remains a large proportion of unexplained heritability in terms of MS risk. Epigenetics can influence the genome without changes to the DNA sequence. Environmental exposures such as smoking and

\footnotetext{
* Correspondence: Jeannette.lechner-scott@hnehealth.nsw.gov.au

${ }^{\dagger}$ Equal contributors

'Centre for Information Based Medicine, Hunter Medical Research Institute,

Newcastle, Australia

${ }^{3}$ School of Medicine and Public Health, Univeristy of Newcastle, Newcastle, Australia

Full list of author information is available at the end of the article
}

vitamin D levels have been demonstrated to modify epigenetic mechanisms, providing a plausible link between environmental factors and disease [2,3]. One such epigenetic mechanism is DNA methylation, which is the addition of a methyl group to CpG dinucleotides. We, and others, have used genome-wide DNA methylation technologies to assess differentially methylated regions (DMRs) of CD4+ T cells in MS patients compared to healthy controls [4-6]. We found a striking methylation signal located on chromosome $6 \mathrm{p} 21$ with a peak signal at HLA-DRB1, which remained after controlling for background SNP effects, as well as 55 non-HLA CpGs that localise to genes previously linked with MS.

In an effort to determine if these previously identified DMRs were specific to CD4+ T cells, we performed a genome-wide methylation study of $\mathrm{CD} 8+\mathrm{T}$ cells using the same cohort, workflow and data analysis as described in our previous study [5]. Briefly, DNA from total CD8+ T cells was extracted from 30 MS patients 
and 28 healthy age- and sex-matched controls. The DNA was bisulphite-converted and hybridised to Illumina $450 \mathrm{~K}$ arrays. Raw fluorescence data were processed using a combination of $\mathrm{R} /$ Bioconductor and custom scripts of a total of 442,672 probes representing individual CpG sites that passed quality control (QC) steps. These CpGs were analysed by statistical modelling of methylation levels ( $\beta$ values) between MS cases and controls.

Figure 1 shows the genome-wide distribution of differential methylation scores for all $\mathrm{CpG}$ sites that passed the nominal $p$ value cut-off of 0.05 . We conducted a stepwise prioritisation strategy to extract the most robust CpG loci associated with MS. Based on the criteria of (i) FDR $p<0.05$ and (ii) $\Delta_{\text {meth }} \geq \pm 0.1$ thresholds, 111 CpGs were extracted. To filter out potential effects of gender and treatment, we performed a subgroup analysis of the methylation statistics as previously described [5]. This process reduced the number of associated CpG sites down to a core panel of 79 (Table 1).

Of the 79 CpGs showing differential methylation in MS patients after filtering, all resided outside the MHC locus on chr 6p21. Of these, 27 were intergenic (34\%), have no gene association, or map to genes of unknown function. Of the remaining 52 loci, $26 \%$ are promoter associated, $9 \%$ are in the $5^{\prime}$ UTR, $5 \%$ are in the 1 st exon, $20 \%$ are in gene bodies and $8 \%$ are in the 3 'UTR. Interestingly, none of these CpGs maps to genes that have previously been reported to have a relationship with MS [7, 8]. There was no overlap between these results and our previous results, and, unlike in CD4+ T cells, there was no gene that contained multiple differentially methylated sites. MORN1 has a single hypermethylated $\mathrm{CpG}$ in both CD4+ and CD8+ T cells; however, it was a different site in each study, making it unlikely that this is a significant finding. Our observations are consistent with the recent study by Bos et al., who also identified minimal overlap between the methylation profiles of CD4+ and CD8+ T cells of MS patients [4].

Using GSEA with WebGestalt, our patient cohort did not have prominent pathways in the KEGG Pathway analysis or disease association analysis. The most significant promoter associated with differential methylation was the ferritin light chain (FTL) gene. The MS cohort displayed decreased methylation at this $\mathrm{CpG}$ locus compared to controls. The gene's biological function is cation transport. One of the statistically significant genes, ERG (ETS-related gene), had a single hypermethylated $\mathrm{CpG}$ in the MS cohort compared to controls. $E R G$ is a member of the transcription factor family involved in activities such as cell proliferation, differentiation,

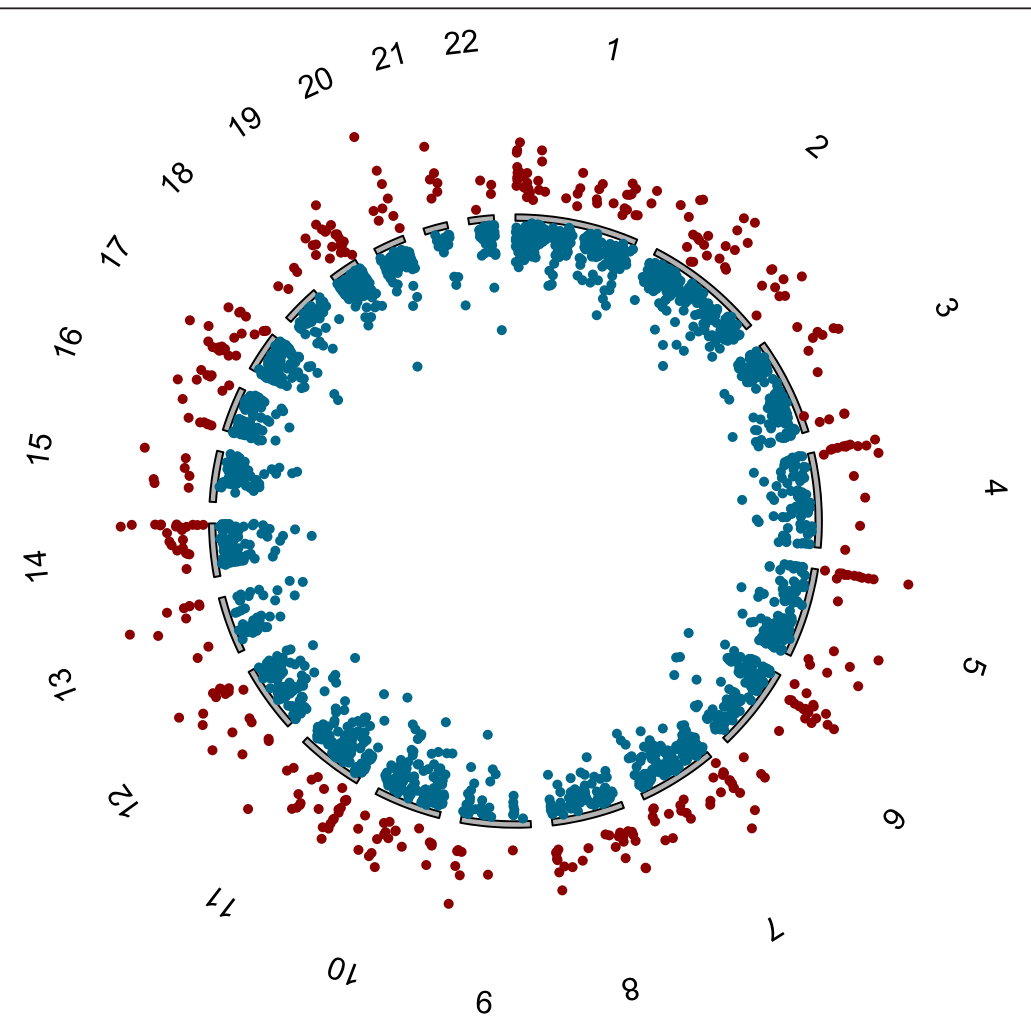

Fig. 1 A genome-wide differential methylation plot based on sites passing a nominal $p$ value of 0.05 . Data points outside the circle represent increased methylation in multiple sclerosis (MS) patients compared to controls (i.e. $\Delta_{\text {meth }}$ ), whereas points inside the circle represent methylation in the MS group 
Table 1 MS-associated CpGs in CD8+ T cells

\begin{tabular}{|c|c|c|c|c|c|c|c|c|}
\hline Probe $I^{a}$ & $\mathrm{CHR}^{\mathrm{b}}$ & Position & Gene $^{c}$ & Feature & Median (case) & Median (control) & $\Delta_{\text {meth }}^{\mathrm{d}}$ & $p$ value $^{\mathrm{e}}$ \\
\hline cg03431738 & 21 & 40031295 & ERG & 5'UTR & 0.81 & 0.68 & 0.13 & 0.004033 \\
\hline cg12026095 & 19 & 49468461 & FTL & TSS200 & 0.30 & 0.49 & -0.18 & 0.004033 \\
\hline cg26228123 & 14 & 73392919 & DCAF4 & TSS200 & 0.09 & 0.20 & -0.11 & 0.004033 \\
\hline cg10478035 & 13 & 80919503 & & - & 0.75 & 0.64 & 0.11 & 0.004033 \\
\hline cg04474988 & 10 & 131770171 & & - & 0.34 & 0.46 & -0.11 & 0.03549 \\
\hline cg25152348 & 22 & 50946712 & $\mathrm{NCAPH} 2$ & 1st exon & 0.30 & 0.47 & -0.17 & 0.03549 \\
\hline cg08206623 & 11 & 2907334 & CDKN1C & TSS1500 & 0.29 & 0.44 & -0.15 & 0.004033 \\
\hline cg13738615 & 9 & 109624741 & ZNF462 & TSS1500 & 0.18 & 0.31 & -0.13 & 0.004033 \\
\hline cg01525244 & 22 & 39548611 & CBX7 & TSS200 & 0.14 & 0.24 & -0.10 & 0.004033 \\
\hline cg12702165 & 12 & 95228136 & MIR492 & TSS200 & 0.65 & 0.54 & 0.11 & 0.004033 \\
\hline cg06443542 & 10 & 100206752 & HPS1 & TSS200 & 0.14 & 0.25 & -0.11 & 0.03549 \\
\hline cg00380172 & 6 & 148663585 & SASH1 & TSS200 & 0.21 & 0.33 & -0.12 & 0.03549 \\
\hline cg19095187 & 6 & 108437051 & & - & 0.17 & 0.31 & -0.14 & 0.03549 \\
\hline cg04488145 & 3 & 46899455 & MYL3 & 3'UTR & 0.83 & 0.73 & 0.11 & 0.03549 \\
\hline cg03027241 & 20 & 49620453 & KCNG1 & 3'UTR & 0.50 & 0.32 & 0.18 & 0.004033 \\
\hline cg11700985 & 10 & 82127205 & DYDC2 & 3'UTR & 0.85 & 0.74 & 0.11 & 0.03549 \\
\hline cg07886142 & 5 & 126793022 & MEGF10 & $3^{\prime}$ UTR & 0.59 & 0.46 & 0.13 & 0.03549 \\
\hline cg18183163 & 2 & 171574141 & SP5 & 3'UTR & 0.12 & 0.26 & -0.14 & 0.03549 \\
\hline cg01181415 & 12 & 16757954 & LMO3 & 5'UTR & 0.22 & 0.36 & -0.14 & 0.03549 \\
\hline cg10143811 & 12 & 16757985 & LMO3 & $5^{\prime} \mathrm{UTR}$ & 0.12 & 0.22 & -0.10 & 0.03549 \\
\hline cg23274123 & 1 & 229478617 & C1orf96 & $5^{\prime}$ UTR & 0.10 & 0.22 & -0.12 & 0.004033 \\
\hline cg00095276 & 5 & 1068111 & SLC12A7 & Body & 0.77 & 0.63 & 0.15 & 0.004033 \\
\hline cg03447557 & 1 & 2273735 & MORN1 & Body & 0.80 & 0.70 & 0.10 & 0.03549 \\
\hline cg02745847 & 17 & 47075880 & IGF2BP1 & Body & 0.17 & 0.31 & -0.13 & 0.03549 \\
\hline cg09406795 & 11 & 64019655 & PLCB3 & Body & 0.25 & 0.38 & -0.13 & 0.000358 \\
\hline cg18016288 & 13 & 95834131 & $\mathrm{ABCC} 4$ & Body & 0.47 & 0.32 & 0.15 & 0.000358 \\
\hline cg14486346 & 2 & 102000131 & CREG2 & Body & 0.78 & 0.66 & 0.12 & 0.03549 \\
\hline cg21937244 & 14 & 103406412 & CDC42BPB & Body & 0.75 & 0.61 & 0.14 & 0.03549 \\
\hline cg11811840 & 2 & 234669166 & UGT1A10 & Body & 0.84 & 0.72 & 0.12 & 0.03549 \\
\hline cg25756617 & 1 & 43734917 & TMEM125 & TSS1500 & 0.69 & 0.58 & 0.11 & 0.03549 \\
\hline cg03768916 & 10 & 49813307 & ARHGAP22 & TSS200 & 0.30 & 0.43 & -0.14 & 0.004033 \\
\hline cg06524757 & 13 & 72441523 & $\mathrm{DACH} 1$ & TSS200 & 0.25 & 0.35 & -0.11 & 0.03549 \\
\hline cg03168749 & 11 & 124413574 & OR8B12 & TSS200 & 0.82 & 0.68 & 0.14 & 0.03549 \\
\hline cg21276022 & 9 & 136390236 & TMEM8C & TSS200 & 0.74 & 0.61 & 0.13 & 0.004033 \\
\hline cg09851596 & 8 & 143545214 & BAl1 & TSS200 & 0.60 & 0.49 & 0.11 & 0.03549 \\
\hline cg25296222 & 11 & 2037173 & & - & 0.76 & 0.65 & 0.11 & 0.03549 \\
\hline cg00878533 & 1 & 2848864 & & - & 0.72 & 0.62 & 0.11 & 0.000358 \\
\hline cg03612700 & 17 & 18970610 & & - & 0.64 & 0.52 & 0.12 & 0.004033 \\
\hline cg03310594 & 7 & 22704316 & & - & 0.82 & 0.69 & 0.13 & $2.34 \mathrm{E}-05$ \\
\hline cg05854694 & 14 & 61123243 & & - & 0.12 & 0.22 & -0.10 & 0.000358 \\
\hline cg12384499 & 15 & 89949617 & & - & 0.19 & 0.31 & -0.11 & 0.004033 \\
\hline cg22509113 & 2 & 91777482 & & - & 0.41 & 0.51 & -0.10 & 0.004033 \\
\hline cg10495084 & 15 & 96889416 & & - & 0.24 & 0.36 & -0.12 & 0.004033 \\
\hline cg18008019 & 13 & 100641646 & & - & 0.10 & 0.23 & -0.12 & 0.03549 \\
\hline
\end{tabular}


Table 1 MS-associated CpGs in CD8+ T cells (Continued)

\begin{tabular}{|c|c|c|c|c|c|c|c|c|}
\hline cg12093775 & 13 & 112548065 & & - & 0.15 & 0.26 & -0.11 & 0.000358 \\
\hline cg12787323 & 10 & 119494959 & & - & 0.16 & 0.27 & -0.11 & 0.004033 \\
\hline cg22792862 & 14 & 67827087 & EIF2S1 & 1st exon & 0.23 & 0.38 & -0.15 & 0.004033 \\
\hline cg08969532 & 10 & 99790438 & CRTAC1 & 1st exon & 0.05 & 0.15 & -0.10 & 0.004033 \\
\hline cg18185028 & 3 & 154042079 & $\mathrm{DH} \times 36$ & 1st exon & 0.30 & 0.41 & -0.11 & 0.000358 \\
\hline cg23059965 & 19 & 50655862 & C19orf41 & $3^{\prime} U T R$ & 0.81 & 0.70 & 0.11 & 0.004033 \\
\hline cg02192678 & 8 & 1495185 & DLGAP2 & $5^{\prime} \cup T R$ & 0.78 & 0.68 & 0.11 & 0.004033 \\
\hline cg02976009 & 6 & 32068226 & TNXB & 5'UTR & 0.71 & 0.59 & 0.12 & 0.03549 \\
\hline cg18073471 & 4 & 81119198 & PRDM8 & $5^{\prime}$ UTR & 0.18 & 0.29 & -0.11 & 0.03549 \\
\hline cg00945810 & 7 & 814391 & HEATR2 & Body & 0.67 & 0.56 & 0.11 & 0.03549 \\
\hline cg04875614 & 4 & 2008706 & WHSC2 & Body & 0.80 & 0.69 & 0.10 & $2.34 \mathrm{E}-05$ \\
\hline cg26920627 & 1 & 7319248 & CAMTA1 & Body & 0.75 & 0.63 & 0.12 & 0.004033 \\
\hline cg26647242 & 2 & 30040525 & ALK & Body & 0.78 & 0.67 & 0.11 & 0.004033 \\
\hline cg04605816 & 20 & 62092443 & KCNQ2 & Body & 0.83 & 0.71 & 0.12 & 0.004033 \\
\hline cg10944063 & 2 & 120233706 & SCTR & Body & 0.58 & 0.46 & 0.12 & 0.004033 \\
\hline cg14595269 & 7 & 151216272 & RHEB & Body & 0.14 & 0.24 & -0.10 & $2.34 \mathrm{E}-05$ \\
\hline cg23720125 & 5 & 177097760 & LOC202181 & Body & 0.85 & 0.73 & 0.12 & 0.004033 \\
\hline cg02047661 & 3 & 51976883 & RRP9 & TSS1500 & 0.64 & 0.52 & 0.11 & 0.004033 \\
\hline cg07925549 & 12 & 52828840 & KRT75 & TSS1500 & 0.75 & 0.63 & 0.12 & 0.03549 \\
\hline cg06697094 & 17 & 54911185 & DGKE & TSS1500 & 0.16 & 0.28 & -0.12 & 0.03549 \\
\hline cg18789663 & 1 & 242688591 & PLD5 & TSS1500 & 0.09 & 0.20 & -0.11 & 0.03549 \\
\hline cg03468541 & 14 & 89029199 & ZC $3 \mathrm{H} 14$ & TSS200 & 0.17 & 0.30 & -0.13 & 0.004033 \\
\hline cg13526221 & 8 & 987389 & & - & 0.79 & 0.69 & 0.11 & 0.004033 \\
\hline cg03313895 & 4 & 24803042 & & - & 0.65 & 0.54 & 0.10 & 0.03549 \\
\hline cg19442593 & 2 & 26252851 & & - & 0.85 & 0.74 & 0.11 & 0.004033 \\
\hline cg04851089 & 6 & 28953923 & & - & 0.39 & 0.54 & -0.15 & 0.004033 \\
\hline cg24520975 & 6 & 31651362 & & - & 0.86 & 0.75 & 0.11 & 0.03549 \\
\hline cg01932076 & 21 & 47394659 & & - & 0.18 & 0.30 & -0.12 & $2.34 \mathrm{E}-05$ \\
\hline cg17555825 & 5 & 76924190 & & - & 0.16 & 0.26 & -0.10 & 0.03549 \\
\hline cg23154781 & 15 & 80634195 & & - & 0.81 & 0.69 & 0.12 & 0.004033 \\
\hline cg00792513 & 6 & 100066698 & & - & 0.34 & 0.47 & -0.14 & 0.03549 \\
\hline cg23708569 & 14 & 106058450 & & - & 0.63 & 0.51 & 0.13 & 2.34E-05 \\
\hline cg09579989 & 12 & 110685438 & & - & 0.81 & 0.71 & 0.10 & 0.03549 \\
\hline cg12077664 & 12 & 125145446 & & - & 0.78 & 0.64 & 0.14 & 0.000358 \\
\hline cg24824082 & 2 & 133030701 & & - & 0.24 & 0.35 & -0.11 & 0.000358 \\
\hline \multicolumn{9}{|c|}{$\begin{array}{l}\text { Dash indicates intergenic } \\
\text { UTR untranslated region, TSS transcription start site } \\
\text { a Probe ID on } 450 \mathrm{~K} \text { chip } \\
{ }^{\mathrm{b}} \text { Chromosome }\end{array}$} \\
\hline
\end{tabular}

apoptosis and inflammation. FTL is a component of ferritin, and defects in this subunit are associated with other neurodegenerative diseases where mutations result in accumulation of iron in the brain [9]. Relapsing-remitting multiple sclerosis (RRMS) patients have increased iron deposits in their grey matter as compared to healthy controls; thus, misregulation of FTL could be important in disease pathology $[10,11]$. Mutations in $D C A F 4$ are associated with leucocyte telomere length, and there is evidence that shortened telomere length in 
leucocytes is associated with other neurodegenerative diseases, such as Parkinson and Alzheimer's disease [12-14]. In addition, one study found a shorted telomere length in primary progressive MS patients, but no correlation between RRMS and differing telomere length has been established [15].

Interestingly, we did not see a cluster of differentially methylated CpGs within $H L A-D R B 1$ as seen in CD4+ T cells [5]. It is well known that the HLA region is notoriously difficult to investigate with many molecular techniques due to increased genetic variation. To minimise the possibility that our observed methylation profile was due to the probes in this region not meeting $\mathrm{QC}$, we used targeted pyrosequencing on available case and control DNA samples. This assay covered seven of the ten differentially methylated CpGs identified in our previous study, but due to high sequence variability, only five of the seven sites returned data. We calculated the median beta values across the five $\mathrm{CpG}$ sites using the $\mathrm{K}-\mathrm{S}$ test. Results showed that the median methylation level in the cases $($ median $=3.6)$ and controls $($ median $=3.6)$ was not significantly different $(p=0.72)$. This supports a conclusion that this MS-related DMR at HLA-DRB1 does not exist in CD8+ T cells but is unique to CD4+ T cells.

A recent study by Bos et al. (2015) also found no major effect loci or clusters of differentially methylated CpGs in the CD8+ T cells of MS patients. However, of the top $40 \mathrm{CpG}$ sites, none overlaps with the top 79 sites found in our study. In addition, we found that approximately half the differentially methylated sites were hypermethylated. This is also in contrast to Bos et al., who found nearly $95 \%$ of sites were hypermethylated in CD8+ T cells. Unlike Bos et al., we chose not to filter out probes that are known to contain SNPs. We reasoned that any false positive signals exclusively due to SNP effects would be subsequently identified by genotyping at the key loci. In support of this notion, pyrosequencing of the key HLA-DRB1 locus did not alter our array-based findings. Additionally, we did not observe a signal at the HLA-DRB1 locus in CD8+ T cells but did in $\mathrm{CD} 4+\mathrm{T}$ cells, providing further support that SNPs are not influencing the findings at this locus.

One important consideration of our study is that the patients were being, or had been, treated with various immunomodulatory therapies at the time of recruitment. In particular, eight patients were being treated with fingolimod, which prevents CD4+ lymphocyte egress from lymphoid tissue. As part of our analysis, we stratified our case-control analysis based on treatment groups in an effort to determine whether overall differential methylation signal may be confounded. None of the patient treatment groups shows a distinct methylation signature, including fingolimod (data not shown), which supports the notion that the small number of treated patients in our cohort is not affecting our results. We do note that this does not necessarily mean that fingolimod is not acting on the methylome, but we can conclude that the small number of patients being treated with fingolimod in our study is not confounding the findings. Future studies will benefit from treatment-naïve patients or will be limiting the study to patients on a particular treatment group.

In this study, we identified 79 CpGs showing minor association with MS. None of these hits was observed in the CD4+ T cells from the same cohort, including the major CD4+ DMR at HLA-DRB1. All genome-wide DNA methylation studies to date have used relatively small sample sizes. This has resulted in identification of large-effect regions only. Large-scale studies are needed to identify minor-effect DMRs. Future studies should also examine the functional consequences of these changes through transcript analysis. Primarily, the results of this study highlight the need to focus on individual cell types when assessing DNA methylation associated with MS susceptibility.

\section{Ethics statement}

The Hunter New England Health Research Ethics Committee and University of Newcastle Human Ethics committee approved this study (05/04/13.09 and H505-0607, respectively). MS patients gave written and verbal consent. The Australian Red Cross Blood Service ethics committee approved the use of blood from healthy donors.

\section{Abbreviations}

DMR: differentially methylated region; DNA: deoxyribonucleic acid; FDR: false discovery rate; GSEA: gene set enrichment assay; GWAS: genome-wide association study; MHC: major histocompatibility complex; MS: multiple sclerosis; QC: quality control; SNP: single nucleotide polymorphism.

\section{Competing interests}

Dr Lechner-Scott's institution receives non-directed funding as well as honoraria for presentations and membership on advisory boards from Sanofi Aventis, Biogen Idec, Bayer Health Care, Merck Serono, Teva and Novartis Australia.

\section{Authors' contributions}

VEM performed experiments, was involved in interpretation of the data, wrote the manuscript and revised all versions of the manuscript. MCG contributed to the original study design, performed experiments, and contributed to the first draft of the manuscript. RAL and MCB performed data analysis, interpretation of the data, and critically reviewed the manuscript. KS performed experiments and critically reviewed the manuscript. LT contributed to initial study design and critically reviewed the manuscript. JLS and RJS initiated and designed the original study, they critically reviewed the manuscript and are responsible for the infrastructure in which in the study was conducted. JLS supervised all aspects of the study. All authors read and approved the final manuscript.

\section{Acknowledgements}

This study was supported by the John Hunter Charitable Trust. Rodney Lea, Vicki Maltby and Katherine Sanders are supported by fellowships from Multiple Sclerosis Research Australia. We would like to thank the MS patients and clinical team at the John Hunter Hospital MS clinic who participated in this study and the Australia Red Cross Blood Service for providing healthy control samples. We also acknowledge the Analytical Biomolecular Research Facility at the University of Newcastle for flow cytometry support, EpigenDx for pyrosequencing and the 
Australian Genome Research Facility for performing the bisulfite conversions and hybridisations to the Illumina 450K arrays.

\section{Author details}

'Centre for Information Based Medicine, Hunter Medical Research Institute, Newcastle, Australia. ${ }^{2}$ School of Biomedical Sciences and Pharmacy, University of Newcastle, Newcastle, Australia. ${ }^{3}$ School of Medicine and Public Health, Univeristy of Newcastle, Newcastle, Australia. ${ }^{4}$ Insitute of Health and Biomedical Innovation, Queensland University of Technology, Brisbane, Australia. ${ }^{5}$ Faculty of Health Sciences and Medicine, Bond University, Gold Coast, Australia. ${ }^{6}$ Division of Molecular Medicine, Pathology North, Newcastle, Australia. ${ }^{7}$ Department of Neurology, Devision of Medicine, John Hunter Hospital, Newcastle, Australia.

Received: 30 August 2015 Accepted: 29 October 2015

Published online: 05 November 2015

\section{References}

1. Broux B, Stinissen P, Hellings N. Which immune cells matter? The immunopathogenesis of multiple sclerosis. Crit Rev Immunol. 2013:33(4):283-306.

2. Wan ES, Qiu W, Baccarelli A, Carey VJ, Bacherman H, Rennard SI, et al. Cigarette smoking behaviors and time since quitting are associated with differential DNA methylation across the human genome. Hum Mol Genet. 2012;21(13):3073-82.

3. Zhu H, Wang X, Shi H, Su S, Harshfield GA, Gutin B, et al. A genome-wide methylation study of severe vitamin D deficiency in African American adolescents. J Pediatr. 2013;162(5):1004-9. e1.

4. Bos SD, Page CM, Andreassen BK, Elboudwarej E, Gustavsen MW, Briggs F, et al. Genome-wide DNA methylation profiles indicate CD8+ T cell hypermethylation in multiple sclerosis. PLoS One. 2015;10(3), e0117403.

5. Graves M, Benton M, Lea R, Boyle M, Tajouri L, Macartney-Coxson D, et al. Methylation differences at the HLA-DRB1 locus in CD4+ T-Cells are associated with multiple sclerosis. Mult Scler. 2013;20(8):1033-41.

6. Baranzini SE, Mudge J, van Velkinburgh JC, Khankhanian P, Khrebtukova I, Miller NA, et al. Genome, epigenome and RNA sequences of monozygotic twins discordant for multiple sclerosis. Nature. 2010;464(7293):1351-6.

7. International Multiple Sclerosis Genetics C, Beecham AH, Patsopoulos NA, Xifara DK, Davis MF, Kemppinen A, et al. Analysis of immune-related loci identifies 48 new susceptibility variants for multiple sclerosis. Nat Genet. 2013:45(11):1353-60.

8. Patsopoulos NA, Barcellos LF, Hintzen RQ, Schaefer C, van Duijn CM, Noble JA et al. Fine-mapping the genetic association of the major histocompatibility complex in multiple sclerosis: HLA and non-HLA effects. PLoS Genet. 2013:9(11), e1003926.

9. Curtis AR, Fey C, Morris CM, Bindoff LA, Ince PG, Chinnery PF, et al. Mutation in the gene encoding ferritin light polypeptide causes dominant adult-onset basal ganglia disease. Nat Genet. 2001;28(4):350-4.

10. Du S, Sah SK, Zeng C, Wang J, Liu Y, Xiong H, et al. Iron deposition in the gray matter in patients with relapse-remitting multiple sclerosis: a longitudinal study using three-dimensional (3D)-enhanced T2*-weighted angiography (ESWAN). Eur J Radiol. 2015;84(7):1325-32.

11. Khalil M, Teunissen C, Langkammer C. Iron and neurodegeneration in multiple sclerosis. Mult Scler Int. 2011;2011:606807.

12. Mangino M, Christiansen L, Stone R, Hunt SC, Horvath K, Eisenberg DT, et al. DCAF4, a novel gene associated with leucocyte telomere length. J Med Genet. 2015;52(3):157-62.

13. Maeda T, Guan JZ, Koyanagi M, Higuchi Y, Makino N. Aging-associated alteration of telomere length and subtelomeric status in female patients with Parkinson's disease. J Neurogenet. 2012;26(2):245-51.

14. Mathur S, Glogowska A, McAvoy E, Righolt C, Rutherford J, Willing C, et al. Three-dimensional quantitative imaging of telomeres in buccal cells identifies mild, moderate, and severe Alzheimer's disease patients. J Alzheimers Dis. 2014;39(1):35-48

15. Guan JZ, Guan WP, Maeda T, Guoqing X, GuangZhi W, Makino N. Patients with multiple sclerosis show increased oxidative stress markers and somatic telomere length shortening. Mol Cell Biochem. 2015;400(1-2):183-7.

\section{Submit your next manuscript to BioMed Central and take full advantage of:}

- Convenient online submission

- Thorough peer review

- No space constraints or color figure charges

- Immediate publication on acceptance

- Inclusion in PubMed, CAS, Scopus and Google Scholar

- Research which is freely available for redistribution

Submit your manuscript at www.biomedcentral.com/submit 